\title{
When the Doors are Closed, a New Window Opens: Prasterone, a New Option in the Treatment of Lichen Sclerosus? A Case Report and Discussion
}

\section{Díaz Ortega Patricia* and García Manero Manuel}

Department of Obstetrics and Gynecology, Hospital García Orcoyen, Estella, Navarra, Spain

*Corresponding Author: Díaz Ortega Patricia, Department of Obstetrics and Gynecology, Hospital García Orcoyen, Estella, Navarra, Spain.
Received: March 09, 2020

Published: May 07, 2020

(C) All rights are reserved by Díaz Ortega

Patricia and García Manero Manuel.

\section{Abstract}

Lichen sclerosus vulvaris is a chronic dermatosis of inflammatory origin, which commonly affects adult, post-menopausal women. It affects the genital and perineal area, where it produces persistent itching, inflammation and pain. Over time, and if treatment is delayed, the scarring that follows the inflammation can produce significant damage such as fusion of the lips, narrowing of the introitus and abolition of the clitoris. All of this can severely affect the quality of life of these women, who are even at increased risk of vulvar cancer.

To date, multiple lines of treatment have been used for this dermatosis, and there is not enough evidence to recommend one over the other.

We present the case of a patient suffering from vulvar lichen sclerosus of long evolution, with little response to various treatments, who was given intravaginal Prasterone, in order to improve her sexual function, which was compromised due to the intense genital atrophy she suffered. From the beginning of the treatment the patient experienced disappearance of the symptoms associated with lichen; and in addition a notable improvement in genital trophism was observed.

We wondered if Prasterone could be a good alternative for the treatment of the symptoms associated with lichen sclerosus, and if it could delay or even prevent the appearance of the trophic disorders associated with it.

Keywords: Lichen Sclerosus; Vulvar Atrophy; Prasterone; DHEA

\section{Abbreviations}

LE: Lichen Sclerosus; DHEA: Dehydroepiandrosterone

\section{Background}

Lichen sclerosus vulvaris, or craurosis vulvaris, is a cicatricial dermatosis of inflammatory origin. The prevalence among the female population is $2 \%$, being more frequent in postmenopausal women. It has a characteristic symptomatology, consisting of intense pruritus, burning sensation and dyspareunia; in severe cases it can significantly affect the quality of life of these patients. Several lines of treatment have been proposed, and although one is clearly recommended over the rest, there is no evidence to date that one treatment is superior to the rest.

We present the case of a patient suffering from ALE of long evolution, with scarce response to various treatments, who was given intravaginal Prasterone in order to improve her sexual function. From the beginning of the treatment the patient experienced disappearance of the symptoms associated with lichen.

\section{Case Report}

We present the case of a postmenopausal patient, being followed for symptoms associated with vulvovaginal atrophy of long evolution and diagnosed with vulvar lichen sclerosus by biopsy and anatomopathological study. The patient suffers from intense vulvar pruritus, which partially subsides with topical corticoid and temporarily with local oestrogens, the symptoms reappearing when the treatment is suspended; she is incapable of maintaining sexual relations and the genital trophism has progressively worsened, presenting a thinned mucosa, with petechiae and abolition of labia minora. The exploration is very painful, and extensive whitish plaques are found in the vulva, compatible with lichen sclerosus, as well as a narrowing of the introitus. It is decided to start treatment with intravaginal Prasterone ova at the recommended dose according to the technical data sheet (6.5 mg per day, intravaginal) in order to improve the patient's sexual function.

After one month of treatment, the patient reports a significant improvement, not only in the level of sexual function, but also in 
the reduction of genital itching and dryness. On examination, a notable improvement in vaginal trophism was observed, as well as a reduction in the extension of the aforementioned whitish plaques (Figure 1).

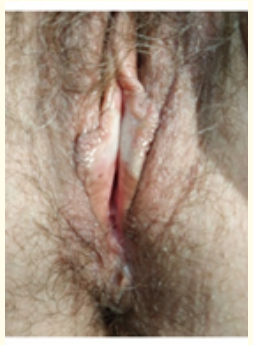

Figure 1: After one month of treatment with Prasterone, a notable improvement in vulvar trophism was observed.

It is decided to continue the treatment, modifying the guideline according to the patient's wishes, to a dose of 3 tablets per week on alternate days, and it is maintained for 4 more months. During the successive visits, the patient refers a clear improvement, having almost completely disappeared the symptomatology associated to the atrophy, and not requiring the use of topical corticoid or other treatments. Vaginal trophism improved significantly and no signs of local or systemic virilization were observed (Figure 2 and 3).

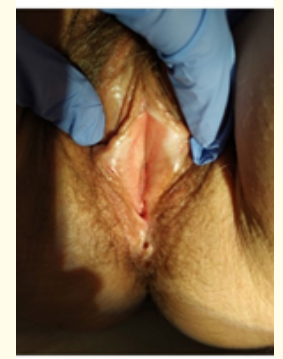

Figure 2: After 2 months of Prasterone treatment.

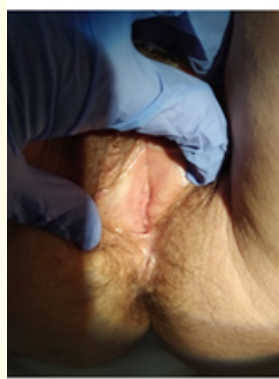

Figure 3: After 4 months of Prasterone treatment.
A vulvar biopsy was performed and reported as lichen sclerosus.

In order to check the action of this medication on the symptoms referred by the patient, it was decided to suspend treatment for one month; shortly after the suspension, the patient referred again to increasing genital itching and stinging. On examination, vaginal trophism had not significantly worsened (Figure 4).

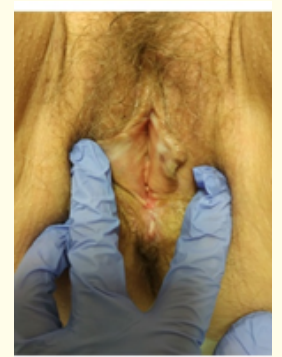

Figure 4: After 1 month of suspension of Prasterone treatment.

\section{Discussion and Conclusion}

Lichen sclerosus vulvaris, or craurosis vulvaris, is a cicatricial dermatosis of inflammatory origin. It is characterized by an inflammatory response dependent on T-lymphocytes (CD4 and CD8) that infiltrate the dermis and release specific Th1-cytokines against targets in the basal layer of the dermis.

The prevalence among the female population is $2 \%$, being more frequent in postmenopausal women. Its main symptom is intense pruritus, followed by burning sensation and dyspareunia; in severe cases it can significantly worsen the quality of life of these patients. In some cases it coexists with other dermatoses, and its clinicopathological evaluation is important when choosing the most appropriate treatment.

Several lines of treatment have been proposed for lichen vulvar, such as corticosteroids, calcineurin inhibitors, topical testosterone and photodynamic therapy. Traditionally, treatment with powerful corticosteroids such as Clovetasol has been considered the most effective treatment; however, this treatment is often accompanied by complications such as skin irritation, secondary infection and skin changes such as telangiectasia.

In a meta-analysis [2] published in 2019, which reviewed 66 studies comparing various treatments for lichen vulva, it was concluded that there is not enough evidence to recommend steroids over other treatment alternatives, as they had similar results to Tacrolimus and Testosterone in terms of remission and recurrence.

In a 2011 Cochrane systematic review [1], seven randomized clinical trials with a total of 249 participants were analyzed, 
comparing various lines of topical treatment for lichen with each other and with placebo; it was found that potent topical steroids achieved a greater decrease in inflammation at the histopathological level, however, there were no significant differences with other treatments in terms of improvement of symptoms, regression and recurrence after stopping treatment. Topical androgens were superior to placebo in reducing symptoms, but not to other treatments. Topical androgens were found to be superior in improving the external appearance of the genitals, with a reduction in atrophy and improved sexual function; however, in prolonged treatment, signs of virilization such as enlargement of the clitoris were observed. This review concludes that more studies would be needed to recommend an alternative over others in the treatment of lichen.

Several studies have demonstrated the role of sex hormones in the physiology of the vagina, and more specifically in vulvovaginal atrophy associated with menopause. The positive effect of androgens, both testosterone and DHEA, on the three layers of the vagina is well known, especially in the deeper layers where they have a stimulating effect on innervation [3,7]. DHEA (dehydroepiandrosterone) is transformed into estrogens and androgens at an intracellular level, producing both effects at the level of the vulvovaginal epithelium, and its beneficial effect and safety at this level is well known when administered intravaginally; improving vaginal trophism and the symptoms derived from atrophy, without producing effects at a systemic level, nor virilization [3-6].

With the presentation of this case, we intend to assess the possible usefulness and effectiveness of prasterone in the treatment of scleroatrophic lichen. Although it is true that histologically there were no changes, there were at the symptomatic level with an almost total disappearance of symptoms such as pruritus, during the administration of the treatment, reappearing after its suspension. Given that according to the latest reviews there is not enough data to recommend one treatment over another traditionally used, and that topical androgens are well known in the treatment of lichen, prasterone could be a good option for these patients. Its easy application and the absence of virilizing effects would be an advantage when assessing a prolonged treatment.

However, more studies comparing prasterone with placebo or other treatments would be needed; as well as comparative studies in terms of dose and duration of treatment.

\section{Authority and Conflict of Interest}

All the authors have participated in all the phases of the elaboration of this manuscript; they also declare that there is no conflict of interests.

\section{Bibliography}

1. Chi CC., et al. "Topical interventions for genital lichen sclerosus". Cochrane Database of Systematic Reviews 12 (2011): CD008240.

2. Perqialiotis V., et al. "An arm-based network meta-analysis on treatments for vulvar lichen sclerosus and a call for development of core outcome sets: Treatment options for vulvar lichen sclerosus". American Journal of Obstetrics and Gynecology (2019).

3. Labrie., et al. "Effect of intravaginal Prasterone on sexual dysfunction in postmenopausal women with vulvovaginal atrophy". Journal of Sexual Medicine 12.12 (2015): 2401-2412.

4. Fernand Labrie., et al. "Is vulvovaginal atrophy due to lack of both estrogens and androgens?". Menopause 24.4 (2017): 452461.

5. Georges Pelletier., et al. "Effects of ovariectomy and dehydroepiandrosterone (DHEA) on vaginal wall thickness and intervation". Journal of Sexual Medicine 9.10 (2012): 2525-2533.

6. Bouchard., et al. "Effect of intravaginal dehydroepiandrosterone (DHEA) on the female sexual function in postmenopausal women: ERC-230 open-label study". Hormone Molecular Biology and Clinical Investigation 25.3 (2016): 181-190.

7. Monica A Pessina., et al. "Differential effects of Estradiol, Progesterone, and Testosterone on vaginal structural integrity". Endocrinology 147.1 (2006): 61-69.

\section{Assets from publication with us}

- Prompt Acknowledgement after receiving the article

- Thorough Double blinded peer review

- Rapid Publication

- Issue of Publication Certificate

- High visibility of your Published work

Website: https://www.actascientific.com/

Submit Article: https://www.actascientific.com/submission.php Email us: editor@actascientific.com Contact us: +919182824667 